\title{
RAMSEY NUMBERS FOR THE PAIR SPARSE GRAPH-PATH OR CYCLE
}

BY

S. A. BURR, P. ERDÖS, R. J. FAUDREE,

C. C. ROUSSEAU AND R. H. SCHELP

\begin{abstract}
Let $G$ be a connected graph on $n$ vertices with no more than $n(1+\varepsilon)$ edges, and $P_{k}$ or $C_{k}$ a path or cycle with $k$ vertices. In this paper we will show that if $n$ is sufficiently large and $\varepsilon$ is sufficiently small then for $k$ odd$$
r\left(G, C_{k}\right)=2 n-1 \text {. }
$$

Also, for $k>2$,

$$
r\left(G, P_{k}\right)=\max \left\{n+[k / 2]-1, n+k-2-\alpha^{\prime}-\delta\right\},
$$

where $\alpha^{\prime}$ is the independence number of an appropriate subgraph of $G$ and $\delta$ is 0 or 1 depending upon $n, k$ and $\alpha^{\prime}$.
\end{abstract}

Introduction. Let $G$ and $H$ be simple graphs. The Ramsey number $r(G, H)$ is the smallest integer $n$ such that for each graph $F$ on $n$ vertices, either $G$ is a subgraph of $F$ or $H$ is a subgraph of $\bar{F}$, the complement of $F$. Calculation of $r(G, H)$ for particular pairs of graphs $G$ and $H$ has received considerable attention, and a survey of such results can be found in [2].

Chvátal [5] proved that if $T_{n}$ is a tree on $n$ vertices and $K_{m}$ is a complete graph on $m$ vertices, then $r\left(T_{n}, K_{m}\right)=(n-1)(m-1)+1$. In [4] it was shown that if $T_{n}$ is replaced by a sparse connected graph $G_{n}$ on $n$ vertices the Ramsey number remains the same (i.e. $\left.r\left(G_{n}, K_{m}\right)=(n-1)(m-1)+1\right)$. For $m=3$ Chvátal's theorem implies $r\left(T_{n}, K_{3}\right)=2 n-1$. In this paper we will show that if $T_{n}$ is replaced by any sparse connected graph $G$ on $n$ vertices and $K_{3}$ is replaced by an odd cycle $C_{k}$, then for appropriate $n$ the Ramsey number is unchanged. In particular we will prove the following.

THEOREM. If $G$ is a connected graph on $n$ vertices and no more than $n(1+\varepsilon)$ edges, then

$$
r\left(G, C_{k}\right)=2 n-1
$$

for $n$ sufficiently large, $\varepsilon$ sufficiently small (both depending upon $k$ ) and $k$ odd.

This theorem falls into a larger category of results considered by Burr [3]. A graph $G$ on $n$ vertices is $H$-good if $r(G, H)=(n-1)(\chi(H)-1)+t(H)$, where $\chi(H)$ is the chromatic number of $H$ and $t(H)$ is the minimum number of vertices in any color class of any $\chi(H)$ vertex coloring of $H$. The above theorem states that $G$ is $C_{k}$-good.

Received by the editors July 1, 1980.

1980 Mathematics Subject Classification. Primary 05C99, 05C35, 05 C55.

() 1982 American Mathematical Society 0002-9947/82/0000-0768/\$04.00 
Gerencsér and Gyárfas [7] proved that $r\left(P_{n}, P_{m}\right)=n+[m / 2]-1$ for $n \geqslant m$, where $P_{k}$ denotes a path with $k$ vertices. Also, Parsons [10] verified for appropriately large $n$ that $r\left(S_{n}, P_{m}\right)=n+m-2-\delta\left(S_{n}\right.$ is a star on $n$ vertices), where $\delta=0$ if $m-1$ divides $n-2$ and $\delta=1$ otherwise. For large $n$ we will generalize these two results by replacing the star and the large path by a sparse graph. The following theorem will be proved.

THEOREM. Let $G$ be a connected graph with $n$ vertices and no more than $n(1+\varepsilon)$ edges. Then for $k \geqslant 2, n$ sufficiently large, and $\varepsilon$ sufficiently small (both depending upon $k$ ),

$$
r\left(G, P_{k}\right)=\max \left\{n+[k / 2]-1, n+k-2-\alpha^{\prime}-\delta\right\},
$$

where $\alpha^{\prime}$ is the independence number of an appropriate subgraph of $G$, and $\delta=0$ if $k-1$ divides $n-2-\alpha^{\prime}$ and $\delta=1$ otherwise.

We now mention some notation that will be used in the paper. Notation not specifically mentioned will follow [9]. The vertex set and edge set of a graph $G$ will be denoted by $V(G)$ and $E(G)$ respectively. The edge with endvertices $u$ and $v$ will be written $u v$. A path with $k$ vertices and endvertices $u$ and $v$ will be denoted by $P_{k}(u, v)$. If the vertices of the path are $\left\{v_{1}, v_{2}, \ldots, v_{k}\right\}$ then this will be expressed by $\left(v_{1}, v_{2}, \ldots, v_{k}\right)$. The corresponding cycle will be written $\left(v_{1}, v_{2}, \ldots, v_{k}, v_{1}\right)$. A path $P$ which is a subgraph of a graph $G$ is a suspended path of $G$ if each vertex of $P$, except for its endvertices, has degree 2 in $G$. If a graph $H$ is isomorphic to a subgraph of $G$, this will many times just be expressed as $H$ is a subgraph of $G$. The graph $G-H$ will be the subgraph of $G$ induced by the vertices of $G$ not in $H$. The graphical parameters $\alpha, \delta$ and $\Delta$ will denote the independence number, minimum degree, and maximum degree of a graph respectively. Also as usual, $\{x\}$ and $[x]$ will denote the least integer greater than or equal to $x$ and the greatest integer less than or equal to $x$ respectively.

Main theorems. There are several facts that are used in the proofs of both of the main theorems as well as in the proofs of other results. Some of these common facts are included in the next lemmas.

LeMMA 1. Let $u$ and $v$ be distinct vertices of a graph $G$ on $s+t$ vertices $(t \geqslant s \geqslant 2)$. Assume $G$ contains a path $P$ which is a $P_{t}(u, v)$ but $G$ contains no $P_{t+1}(u, v)$.

(i) If $t \geqslant 2 s$, then $\bar{G}$ contains a $P_{2\{s / 2\}+1}$.

(ii) If $t \geqslant 5 s-1$, then $\bar{G}$ contains $a P_{2 s+1}$.

(iii) If $t \geqslant 5 s-1$, then $\bar{G}$ contains a $P_{2 s-1}$ between any pair of vertices not in $P$ or $\bar{G}$ contains $a K_{2 s+1}$.

Proof. Denote the path $P$ by $\left(x_{1}, x_{2}, \ldots, x_{t}\right)$ and let $S$ be the set of vertices of $G$ not in $P$. Since the path $P$ cannot be lengthened, no vertex of $S$ is adjacent in $G$ to two consecutive vertices of $P$. Thus for any pair $\left\{x_{i}, x_{i+1}\right\}$ of consecutive vertices of $P$, two of any three vertices of $S$ are commonly adjacent in $\bar{G}$ to either $x_{i}$ or $x_{i+1}$. 
(i) Since $t \geqslant 2 s$ repeated application of the above fact implies that there exist two vertex disjoint paths $Q_{1}$ and $Q_{2}$ satisfying the following.

(a) Their first and last vertices are in $S$.

(b) Their vertices alternate between $S$ and $P$.

(c) $S \subseteq V\left(Q_{1}\right) \cup V\left(Q_{2}\right)$.

With no loss of generality we can assume that $Q_{1}$ contains at least $\{s / 2\}$ vertices of $S$. If $Q_{1}$ contains at least $\{s / 2\}+1$ vertices, then $Q_{1}$ is the desired path. If $Q_{1}$ contains precisely $\{s / 2\}$ vertices of $S$ (and $\{s / 2\}-1$ vertices of $P$ ), then $Q_{1}$ can be extended to a $P_{2\{s / 2\}+1}$ by adjoining a vertex from $P$ to each end of $Q_{1}$. This is possible since each vertex of $S$ is adjacent in $\bar{G}$ to at least $s$ vertices.

(ii) and (iii). If a vertex $w$ of $S$ is adjacent in $G$ to $x_{i}$ and $x_{j}$ of $P(i<j<t)$, then $x_{i+1} x_{j+1}$ is in $\bar{G}$. Otherwise, $\left(x_{1}, \ldots, x_{i}, w, x_{j}, x_{j-1}, \ldots, x_{i+1}, x_{j+1}, \ldots, x_{t}\right)$ is a $P_{t+1}(u, v)$. Therefore if a vertex $w$ of $S$ is adjacent in $G$ to $2 s+1$ vertices of $P$, the $2 s$ vertices which are successors (the last vertex may not have a successor) of these vertices along $P$ together with $w$ form a $K_{2 s+1}$ in $\bar{G}$. We can thus assume that each vertex of $S$ is adjacent in $G$ to at most $2 s$ vertices of $P$. This implies that each pair of vertices of $S$ are commonly adjacent in $\bar{G}$ to at least $s-1$ vertices of $P$. Hence for any pair of vertices $w_{1}$ and $w_{2}$ of $S$ there exists a $P_{2 s-1}\left(w_{1}, w_{2}\right)$ in $\bar{G}$ such that the vertices alternate between $S$ and $P$. This path can be extended to a $P_{2 s+1}$ since each of $w_{1}$ and $w_{2}$ are adjacent in $\bar{G}$ to at least $3 s-1$ vertices of $P$.

LEMMA 2. Let $G$ be a graph on $n$ vertices and $n+t$ edges. If $G$ has no isolated vertices and no suspended path with more than $s$ vertices, then $G$ has at least $\{n /(2 s)-3 t / 2\}$ vertices of degree 1 .

Proof. Let $H$ be the graph (possibly multigraph) obtained from $G$ by replacing each suspended path of $G$ by an edge. Thus if $H$ has $m$ vertices, then $H$ has $m+t$ edges and every vertex has degree 1 or at least 3 . If $x$ is the number of vertices in $G$ (and in $H$ ) of degree 1 , then

$$
x+3(m-x) \leqslant 2(m+t) .
$$

Since $G$ has no suspended path with more than $s$ vertices, $G$ can be obtained from $H$ by replacing each edge of $H$ by a suspended of appropriate length less than or equal to $s$. Thus

$$
n \leqslant s(m+t)
$$

Therefore the two displayed equations imply

$$
x \geqslant n / 2 s-3 t / 2
$$

which completes the proof.

LEMMA 3. Let $m \geqslant n, n_{1} \geqslant n_{2} \geqslant \cdots \geqslant n_{l} \geqslant 1, k \geqslant 3$ and $l \geqslant\{k / 2\}-1$ be positive integers. Let $H$ be a graph on $n-\sum_{i=1}^{l} n_{i}$ vertices with $l$ distinct vertices designated $v_{1}, v_{2}, \ldots, v_{l}$, and $G$ a graph on $n$ vertices obtained from $H$ by adjoining for each $i(1 \leqslant i \leqslant l)$, a star with $n_{i}$ edges and center at $v_{i}$. If

$$
r\left(H, P_{k}\right) \leqslant m-\left(\sum_{i=1}^{\{k / 2\}-1} n_{i}\right)(k-1),
$$


then

$$
r\left(G, P_{k}\right) \leqslant m+[k / 2]-1 .
$$

Proof. Let $F$ be a graph on $m+[k / 2]-1$ vertices. Assume that $F$ does not have $G$ as a subgraph and $\bar{F}$ does not contain a $P_{k}$. We will show that this leads to a contradiction.

Select a path of maximal length in $\bar{F}$. By assumption this path has at most $k-1$ vertices and the endvertices of the path are adjacent in $F$ to all vertices not on the path. Delete the vertices of this path and then repeat this procedure a total of $\left(\sum_{i=1}^{\{k / 2\}-1} n_{i}\right)-1$ times. This leaves a graph $F^{\prime}$ with at least $m-\left(\sum_{i=1}^{\{k / 2\}-1} n_{i}\right)$ $\cdot(k-1)$ vertices. Thus $F^{\prime}$ contains $H$ as a subgraph. Let $A$ be the set of endvertices of the paths deleted. Hence $|A| \geqslant \sum_{i=1}^{\{k / 2\}-1} n_{i}$.

The graph $F$ would contain $G$ as a subgraph if there were disjoint sets of vertices $S_{1}, S_{2}, \ldots, S_{l}\left(\left|S_{i}\right|=n_{i}\right)$ in $F$ but not in $H$ such that each vertex of $S_{i}$ is adjacent in $F$ to the vertex $v_{i}$ for $1 \leqslant i \leqslant l$. Since $F$ does not contain $G$ as a subgraph, Hall's theorem [8] implies that there exists a set $L=\left\{v_{i_{1}}, v_{i_{2}}, \ldots, v_{i_{r}}\right\}$ such that

$$
|N \cap V(F-H)|<\sum_{j=1}^{r} n_{i},
$$

where $N$ is the set of vertices which is adjacent in $F$ to at least one of $v_{i j}, 1 \leqslant j \leqslant r$. Clearly then, $|N \cup V(H)|<|V(H)|+\sum_{j=1}^{r} n_{i_{j}} \leqslant n$. Therefore $\bar{F}$ contains a complete bipartite graph $K_{r,[k / 2]}$. Also each vertex of $A$ is adjacent in $F$ to each $v_{i}$, $1 \leqslant i \leqslant l$. Since $|A| \geqslant \sum_{i=1}^{\{k / 2\}} n_{i}$ and $A \cap V(H)=\varnothing, r \geqslant\{k / 2\}$. This implies that $\bar{F}$ contains a $P_{k}$, a contradiction.

The next lemma will be needed in the inductive step of the proof of Proposition 5. The graph $G+e$ is a graph obtained from the graph $G$ by adding the edge $e$ between two nonadjacent vertices of $G$.

LEMMA 4. For any graph $G$ and for $k \geqslant 2$,

$$
r\left(G+e, P_{k}\right) \leqslant r\left(G, P_{k}\right)+k-1 .
$$

Proof. Let $F$ be a graph on $r\left(G, P_{k}\right)+k-1$ vertices and assume that $\bar{F}$ does not contain a $P_{k}$. Select a path of maximal length in $\bar{F}$ and let $w$ be an endvertex of this path. Since $\bar{F}$ contains no $P_{k}$, the set $S$ consisting of $w$ and the vertices adjacent to $w$ in $\bar{F}$ has at most $k-1$ vertices. Therefore there is a copy of $G$ in $F$ which is vertex-disjoint from $S$. Let $u$ and $v$ be the endvertices in this copy of $G$ of the edge $e$. Since $w$ is adjacent in $F$ to each vertex of this copy of $G$, replacing $u$ (or $v$ ) by $w$ gives a copy of $G+e$.

Let $G$ be a connected graph on $n$ vertices and no more than $[n(1+\varepsilon)]$ edges. If $n$ is sufficiently large and $\varepsilon$ sufficiently small, then $G$ has either a long suspended path or a large number of vertices of degree 1 . This fact will be used in the proof of the following proposition. The various cases appearing in the proof are also a consequence of this fact.

Proposition 5. For integers $n, k \geqslant 1$, let $G$ be a connected graph with $n$ vertices and no more than $[n(1+1 / 18 k)]$ edges. Then $r\left(G, P_{k}\right)<n+13 k^{5}$. 
Proof. The result is trivial for $k=1$ or 2 , so assume $k>3$. The proof will be by induction on $n$. Since $r\left(K_{n}, P_{k}\right)<n k$, [5], the result is true if $n \leqslant 13 k^{4}$. Thus assume $n>13 k^{4}$ and the result is true for all appropriate graphs with less than $n$ vertices. Let $F$ be a graph on $n+13 k^{5}$ vertices. We will assume that $\bar{F}$ contains no $P_{k}$ and show that $G$ is a subgraph of $F$.

Case I. $G$ has a suspended path with $3 k-3$ vertices.

Let $H$ be the graph on $n=k+1$ vertices obtained from $G$ by shortening the suspended path by $k-1$ vertices. Therefore $H$ has at most $[n(1+(1 / 18 k))]-k$ $+1 \leqslant[(n-k+1)(1+1 / 18 k)]+1$ edges. The induction assumption and Lemma 4 imply that $F$ contains $H$ as a subgraph. Of course $G$ can be obtained from $H$ by lengthening the suspended path in $H$ by $k-1$ vertices. Let $H^{\prime}$ be a subgraph of $F$ in which this suspended path has been lengthened as much as possible (up to $k-1$ ). If $H^{\prime}$ is isomorphic to $G$, the proof of this case is complete. If not, then select a set $S$ of $k-1$ vertices of $F$ not in $H^{\prime}$. Let $F^{\prime}$ be the subgraph of $F$ spanned by the vertices of the suspended path of $H^{\prime}$ and the set $S$. Lemma 1(i) implies that $\bar{F}^{\prime}$, and hence $\bar{F}$, contains a $P_{k}$. This contradiction completes the proof of this case.

Case II. $G$ has a vertex adjacent to at least $2 k-2$ vertices of degree 1 .

Let $S$ be a set of $2 k-2$ vertices of degree 1 in $G$ which are adjacent in $G$ to a vertex $v$. Let $u$ be the endvertex of a path $P$ of maximal length in $\bar{F}$. The path $P$ has at most $k-1$ vertices. If $H=G-S$, then $H$ has $n-2 k+2$ vertices and at most $[(n-2 k+2)(1+1 / 18 k)]+1$ edges. The induction assumption and Lemma 4 imply that $r\left(H, P_{k}\right) \leqslant n-k+1+13 k^{5}$. Therefore $F$ contains $H$ as a subgraph such that $H$ is vertex-disjoint from $P$. Since $u$ is adjacent in $F$ to each vertex of $F$ not on $P$, replacing $v$ in $H$ by $u$ gives a copy of $G$ in $F$.

Case III. $G$ has no suspended path with $3 k-3$ vertices and no vertex adjacent to $2 k-2$ vertices of degree 1 .

Lemma 2 implies that $G$ has at least $n /(2(3 k-3))-3 n / 36 k \geqslant n / 12 k$ vertices of degree 1 . Since $n>13 k^{4}, G$ has at least $\left\{\frac{13}{12} k^{3}\right\}$ vertices of degree 1 . The fact that no vertex of $G$ is adjacent to more than $2 k-3$ vertices of degree 1 insures that there are distinct vertices $v_{1}, v_{2}, \ldots, v_{l},\left(l \geqslant 13 k^{3} /(12(2 k-3))\right)$ in $G$ with each $v_{i}$ adjacent to $n_{i}$ vertices of degree 1 . In fact we can assume that $2 k-2>n_{1}$ $>n_{2}>\cdots \geqslant n_{l} \geqslant 1$ and $\left\{\frac{13}{12} k^{3}\right\}=\sum_{i=1}^{l} n_{i}$. Let $H$ be the graph obtained from $G$ by deleting those $t=\sum_{i=1}^{l} n_{i}$ vertices of degree 1 . The graph $H$ has $n-t$ vertices and at most $[(n-t)(1+1 / 18 k+t / 18 k)]$ edges. The induction assumption and Lemma 4 imply that

$$
r\left(H, P_{k}\right) \leqslant n-t+13 k^{5}+\{t / 18 k\}(k-1) .
$$

A straightforward calculation implies that

$$
r\left(H, P_{k}\right) \leqslant\left(n+13 k^{5}-([k / 2]-1)\right)-\left(\sum_{i=1}^{\{k / 2\}-1} n_{i}\right)(k-1) .
$$

Therefore by Lemma 3,

$$
r\left(G, P_{k}\right) \leqslant n+13 k^{5}
$$


Proposition 5 will be used heavily in the proof of the following theorem. Also the outlines of the two proofs are similar in that they both use the structure of a sparse connected graph. Some additional notation is needed before the theorem can be stated.

If $v$ is a vertex of a graph $G$, let $G_{v}$ be the subgraph of $G$ induced by the vertices distinct from $v$ and not adjacent to $v$. Let $\alpha^{\prime}(G)=\min \left\{\alpha\left(G_{v}\right): v \in V(G)\right\}$.

THEOREM 6. Let $G$ be a connected graph with $n$ vertices and no more than $\left[n\left(1+1 / 81 k^{5}\right)\right]$ edges. Then for $k \geqslant 2$ and $n \geqslant 352 k^{12}$,

$$
r\left(G, P_{k}\right)=\max \left\{n+[k / 2]-1, n+k-2-\alpha^{\prime}(G)-\delta\right\},
$$

where $\delta=0$ if $k-1$ divides $n+k-3-\alpha^{\prime}(G)$ and $\delta=1$ otherwise.

Note that if $\alpha^{\prime}(G)$ is as large as $\{k / 2\}$, then Theorem 6 implies $r\left(G, P_{k}\right)=n+$ $[k / 2]-1$. Also, unless $G$ has a vertex of very large degree, $\alpha^{\prime}(G) \geqslant k$. For example, if $v$ is a vertex of degree $<n\left(1-1 / 81 k^{5}\right)$, then $G_{v}$ has $t>n / 81 k^{5}$ vertices and at most $t+n / 81 k^{5}$ edges. Therefore $G_{v}$ has average degree no more than 4. Thus Turán's Theorem implies that $G_{v}$ has independence number at least $t / 5 \geqslant n / 405 k^{5}>k$. Thus the only time it will be necessary to calculate $\alpha^{\prime}(G)$ will be when $G$ has a unique vertex $v$ of very large degree, and in this case $\alpha^{\prime}(G)$ is just the independence number of $G_{v}$.

Proof (Theorem 6). The graph $K_{n-1} \cup K_{[k / 2]-1}$ has no connected subgraph with $n$ vertices and its complement has no $P_{k}$. Let $n+k-3-\alpha^{\prime}(G)-\delta=$ $t(k-1)+s$, where $0<s \leqslant k-1$. Note that

$$
n+k-3-\alpha^{\prime}(G)-\delta=(t-k+2+s)(k-1)+(k-1-s)(k-2) \text {. }
$$

Let $L$ be the graph whose complement is the graph

$$
(t-k+2+s) K_{k-1} \cup(k-1-s) K_{k-2} \text {. }
$$

Clearly $\bar{L}$ contains no $P_{k}$. We will show that $L$ does not contain $G$ as a subgraph if $\alpha^{\prime}(G) \leqslant k$. Assume $G$ is a subgraph of $L$ and let $v$ be the unique vertex of maximal degree in $G$. The vertex $v$ is in one of the disjoint independent sets of $L$, say $S$. The set $S$ has at least $k-1-\delta$ vertices. Any other vertices of $G$ in $S$ must be independent and not adjacent to $v$. Therefore $S$ contains at most $\alpha^{\prime}(G)+1$ vertices of $G$. Hence $L$ must have at least

$$
n+(k-1-\delta)-\left(1+\alpha^{\prime}(G)\right)=n+k-2-\alpha^{\prime}(G)-\delta
$$

vertices, a contradiction. The above two examples give the lower bound for $r\left(G, P_{k}\right)$.

The proof of the upper bound for $r\left(G, P_{k}\right)$ will be broken into three cases, just as the proof of Proposition 5. Let $F$ be a graph on

$$
\max \left\{n+[k / 2]-1, n+k-2-\alpha^{\prime}(G)-\delta\right\}
$$

vertices whose complement $\bar{F}$ has no $P_{k}$. We will show that $G$ is a subgraph of $F$. The result is trivial for $k=2$, so assume $k \geqslant 3$.

Case I. $G$ has a suspended path with at least $13 k^{5}+3 k$ vertices.

Let $H$ be the graph on $n-13 k^{5}$ vertices obtained from $G$ by shortening the suspended path by $13 k^{5}$ vertices. By Proposition $5, F$ has $H$ as a subgraph. Let $H^{\prime}$ 
be a subgraph of $F$ with a maximal number of vertices which can be obtained from $H$ by lengthening the suspended path as much as possible (up to $13 k^{5}$ ). If $H^{\prime}$ is $G$, the proof is complete. If not, Lemma 1(ii), applied to the subgraph of $F$ induced by the vertices of the suspended path of $H^{\prime}$ along with [k/2] vertices not in $H^{\prime}$, implies that $\bar{F}$ contains a $P_{k}$. This contradiction completes the proof of this case.

Case II. $G$ has a vertex adjacent to at least $13 k^{5}+k$ vertices of degree 1 .

Let $S$ be a set of $13 k^{5}+k$ vertices of degree 1 adjacent in $G$ to a vertex $v$ and let $H=G-S$. Thus $H$ has $n-13 k^{5}-k$ vertices and $r\left(H, P_{k}\right) \leqslant n-k$ by Proposition 5.

Consider a maximal length path $P=\left(x_{1}, x_{2}, \ldots, x_{t}\right)$ in $\bar{F}$. By assumption $t<k$. If there exists an $i(1<i \leqslant t)$ such that $x_{1} x_{i}$ and $x_{i-1} x_{t}$ are edges in $\bar{F}$, then the vertices of $P$ form a cycle (i.e. $\left(x_{1}, x_{i}, x_{i+1}, \ldots, x_{t}, x_{i-1}, x_{i-2}, \ldots, x_{2}, x_{1}\right)$ is a $\left.C_{t}\right)$. The maximality of the length of $P$ implies that the vertices of $P$ are a component of $\bar{F}$. If no such $i$ exists, then the sum of the degrees in $\bar{F}$ of $x_{1}$ and $x_{t}$ is $\leqslant t-1$. This would insure that $\bar{F}$ has a vertex of degree $\leqslant[k / 2]-1$. Therefore, without loss of generality we can assume that $\bar{F}$ is the disjoint union of complete graphs each with less than $k$ vertices or that $\bar{F}$ has a vertex of degree $\leqslant[k / 2]-1$.

Let $u$ be a vertex of $\bar{F}$ of minimum degree, and let $N$ be the vertices of $\bar{F}$ adjacent to $u$. Then $N \cup\{u\}$ has at most $k-1$ vertices. Since $r\left(H, P_{k}\right) \leqslant n-k$, $F$ has a subgraph $H$ which is vertex-disjoint from $N \cup\{u\}$.

If $u$ has degree at most [k/2] -1 , then replace the vertex $v$ in $H$ by the vertex $u$. Since $u$ is adjacent in $F$ to each vertex of $H$, this gives another copy of $H$ in $F$. Also, since $u$ is adjacent in $F$ to all but possibly [k/2] -1 vertices, this verifies that $G$ is a subgraph of $F$.

Assume $u$ has degree at least $[k / 2]$. In this case $\bar{F}$ is the disjoint union of complete graphs each with at most $k-1$ vertices. We have that $u$ is in some component $C$ of $\bar{F}$ with $t \leqslant k-1$ vertices. In fact, if $F$ has $n+k-2-\alpha^{\prime}(G)-$ $\delta$ vertices, then $t \leqslant k-1-\delta$. Since $r\left(H, P_{k}\right) \leqslant n-k, F$ has a subgraph $H$ which is vertex-disjoint from $C$. Replace the vertex $v$ of $H$ by $u$. This gives a copy of $H$ in $F$, which we will denote by $H^{\prime}$. The graph $H^{\prime}$ has only the vertex $u$ in common with $C$. Select an independent set $B$ of $\min \left\{\alpha\left(H_{u}^{\prime}\right), t-1\right\}$ vertices in $H_{u}^{\prime}$. Obtain another copy of $H$, which we will denote by $H^{\prime \prime}$, by replacing the vertices $B$ of $H^{\prime}$ with $|B|$ vertices of $C$ distinct from $u$. This can be done, since each vertex of $C$ is adjacent in $F$ to each vertex of $H^{\prime}$ except $u$. Since $\alpha\left(H_{u}^{\prime}\right)=\alpha\left(H_{v}\right)$ and $\alpha\left(G_{v}\right) \geqslant$ $\alpha^{\prime}(G), u$ is adjacent in $F$ to all except at most $t-1-\alpha^{\prime}(G)$ of the vertices in $F-H^{\prime \prime}$. Therefore the vertex $u$ is adjacent in $F$ to at least $13 k^{5}+k$ vertices not in $H^{\prime \prime}$. Hence $F$ contains $G$ as a subgraph.

Case III. $G$ has no suspended path with $13 k^{5}+3 k$ vertices and no vertex adjacent to $13 k^{5}+k$ vertices of degree 1 .

Lemma 2 implies that $G$ has at least

$$
n /\left(2\left(13 k^{5}+3 k\right)\right)-3 n /\left(2 \cdot 81 k^{5}\right) \geqslant\left(n / k^{5}\right)\left(\frac{1}{27}-\frac{1}{54}\right)=n / 54 k^{5}
$$

vertices of degree 1 . Since $n \geqslant 352 k^{12}, G$ has at least $\left(\frac{352}{54}\right) k^{7}$ vertices of degree 1 . Thus there exist vertices $v_{1}, v_{2}, \ldots, v_{l}$ in $G\left(l \geqslant\left(\frac{352}{54}\right) k^{7} /\left(13 k^{5}+k\right)\right)$ such that $v_{i}$ is 
adjacent to $n_{i}$ vertices of degree 1 with $13 k^{5}+k>n_{1} \geqslant n_{2} \geqslant \cdots \geqslant n_{l} \geqslant 1$. We can also assume that $\left\{\left(\frac{352}{54}\right) k^{7}\right\}=\sum_{i=1}^{l} n_{i}$.

Let $H$ be the graph obtained from $G$ by deleting these $t=\sum_{i=1}^{l} n_{i}$ vertices of degree 1. Therefore $H$ has $n-t$ vertices and at most $\left[(n-t)\left(1+1 / 81 k^{5}\right)+\right.$ $\left(t / 81 k^{5}\right)$ ] edges. Thus Proposition 5 and Lemma 4 imply

$$
r\left(H, P_{k}\right) \leqslant n-t+13 k^{5}+\left[\frac{t}{81 k} 5\right](k-1) .
$$

Using very bad approximations, direct calculations verify that

$$
r\left(H, P_{k}\right) \leqslant n-\left(\sum_{i=1}^{\{k / 2\}-1} n_{i}\right)(k-1)
$$

Therefore by Lemma $3, G$ is a subgraph of $F$. This completes the proof of the theorem.

The lower bound on $n$, the number of vertices in $G$ in Theorem 6, and the upper bound on the number of edges in $G$ in Proposition 5 and Theorem 6 are by no means sharp. Sometimes to simplify the calculations slightly weaker bounds were used, but significant improvement in these bounds will necessitate different methods of proof.

There are several interesting corollaries of Theorem 6. The first one is a consequence of the remarks made prior to the proof of Theorem 6 , and the last three are a result of calculating $\alpha^{\prime}(G)$. The last two are special cases of well-known results.

COROllary 7. Let $G$ be a connected graph with $n$ vertices and no more than $\left[n\left(1+1 / 81 k^{5}\right)\right]$ edges. If $\Delta(G) \leqslant n\left(1-1 / 81 k^{5}\right), n \geqslant 352 k^{12}$ and $k \geqslant 2$, then

$$
r\left(G, P_{k}\right)=n+[k / 2]-1 \text {. }
$$

CoRollaRy 8. If $T_{n}$ is a tree on $n$ vertices with $\Delta\left(T_{n}\right)<n-k, n \geqslant 352 k^{12}$ and $k \geqslant 2$, then

$$
r\left(T_{n}, P_{k}\right)=n+[k / 2]-1
$$

COROLlaRy $9[6,7]$. If $n \geqslant 352 k^{12}$ and $k \geqslant 2$, then

(i) $r\left(P_{n}, P_{k}\right)=n+[k / 2]-1$,

(ii) $r\left(C_{n}, P_{k}\right)=n+[k / 2]-1$.

In the results as stated in both [6] and [7] the restrictions on $n$ are only linear in $k$.

COROLlaRY $10[\mathbf{1 0}]$. If $n \geqslant 352 k^{12}$ and $k \geqslant 2$, then

$$
r\left(S_{n}, P_{k}\right)=n+k-2-\delta \text {, }
$$

where $\delta=0$ if $k-1$ divides $n-2$ and $\delta=1$ otherwise.

In the result stated in [10] the restriction on $n$ is only quadratic in $k$.

We will use the results on $r\left(G, P_{k}\right)$ to obtain the numbers $r\left(G, C_{k}\right)$ for $k$ odd. Two additional lemmas will be needed. These we will state and prove now. 
LEMMA 11. Let $G$ be a graph with $n$ vertices and minimum degree $\delta \geqslant 1$. If $v$ is a vertex of degree $\delta$, then

$$
r\left(G, C_{k}\right) \leqslant \max \left(r\left(G-v, C_{k}\right), \delta r\left(G, P_{k-1}\right)+n-\delta\right) .
$$

Proof. Let $F$ be a graph with $\max \left(r\left(G-v, C_{k}\right), \delta r\left(G, P_{k-1}\right)+n-\delta\right)$ vertices whose complement $\bar{F}$ does not contain a $C_{k}$. Therefore by assumption, $G-v$ is a subgraph of $F$. Let $N$ be the vertices of $G-v$ which are adjacent in $G$ to $v$, and let $S$ be the vertices of $F$ not in $G-v$. Thus $N$ has $\delta$ vertices and $S$ has at least $\delta\left(r\left(G, P_{k-1}\right)-1\right)+1$ vertices. If $F$ does not contain $G$ as a subgraph, then each vertex of $S$ must be adjacent in $\bar{F}$ to at least one vertex of $N$. Therefore some vertex $w$ of $N$ has degree at least $r\left(G, P_{k-1}\right)$ in $\bar{F}$. This implies that either $F$ contains $G$ as a subgraph or there is a $C_{k}$ in $\bar{F}$ containing $w$. This completes the proof.

LEMMA 12. If $G$ is a graph with $n$ vertices and l edges, then

$$
r\left(G, C_{k}\right) \leqslant n+2 l k-2 l / n \text {. }
$$

PROOF. The proof will be by induction on $n$. If $n=1$, then $l=0$ and clearly $r\left(G, C_{k}\right) \leqslant 1$. Proceed by induction and let $v$ be a vertex of minimal degree $\delta$ in $G$; Lemma 11 implies

$$
r\left(G, C_{k}\right) \leqslant \max \left(r\left(G-v, C_{k}\right), \delta \cdot r\left(G, P_{k-1}\right)+n-\delta\right) .
$$

The induction assumption and the fact that $r\left(K_{n}, P_{k}\right) \leqslant n k$ give that

$$
r\left(G, C_{k}\right) \leqslant \max \left(n-1+2 k(l-\delta)-\frac{2(l-\delta)}{n-1}, \delta k n+n-\delta\right) .
$$

Using that $\delta \leqslant 2 l / n$, it is easily verified that $\delta k n+n-\delta \leqslant n+2 k l-2 l / n$. Therefore direct calculation gives

$$
r\left(G, C_{k}\right) \leqslant n+2 k l-\frac{2 l}{n}
$$

which completes the proof.

Note that Lemma 12 implies for example that $r\left(K_{n}, C_{k}\right) \leqslant n+2 n(n-1) k / 2 \leqslant$ $n^{2} k[1]$.

Proposition 13. Let $G$ be a connected graph with $n$ vertices and no more than $\left[n\left(1+1 / 12 k^{2}\right)\right]$ edges. For all $n, k \geqslant 3$,

$$
r\left(G, C_{k}\right) \leqslant 2 n+13 k^{5} \text {. }
$$

Proof. Assume the proposition is not true. Then there exists a graph $F$ on $2 n+13 k^{5}$ vertices which does not have $G$ as a subgraph and whose complement $\bar{F}$ does not contain a $C_{k}$. Let $L$ be the graph obtained from $G$ by successively deleting vertices of degree 1 and shortening suspended paths with at least $5[(k+1) / 2]+1$ vertices by a vertex. We will show that $L$ is also not a subgraph of $F$. This will lead to a contradiction.

Case I. $G$ has a suspended path with at least $5[(k+1) / 2]+1$ vertices.

Let $H$ be the graph obtained from $G$ by shortening the suspended path by a vertex. If $F$ contains $H$ as a subgraph, then Lemma 1 implies that for any pair of 
vertices of $F$ not in $H$ there is a path with $k$ ( $k$ odd) or $k-1$ ( $k$ even) vertices between them. This path can be assumed to use any $[(k+1) / 2]$ predetermined vertices of $F$ not in $H$. Since $r\left(G, P_{k}\right) \leqslant n+13 k^{5}$ by Proposition 5 , either $F$ contains $G$ as a subgraph or $\bar{F}$ contains a $C_{k}$ using the path described earlier. We can thus conclude that $H$ is not a subgraph of $F$.

Case II. $G$ has vertex of degree 1 .

Let $H$ be the graph obtained from $G$ by deleting a vertex of degree 1 . Let $v$ be the vertex of $G$ adjacent to this vertex of degree 1 . If $F$ contains $H$ as a subgraph, then the vertex $v$ is adjacent in $\vec{F}$ to at least $n+13 k^{5}+1$ vertices. Since $r\left(G, P_{k}\right) \leqslant n+13 k^{5}$, we can assume that $F$ does not contain $H$ as a subgraph.

Repeated application of the situations in cases 1 and 2 imply that $L$ is not a subgraph of $F$. The graph $L$ has $l$ vertices, no more than $l+\left[n / 12 k^{2}\right]$ edges, no suspended path with more than $5[(k+1) / 2]$ vertices, and no vertices of degree 1 . Lemma 2 implies that $l$ satisfies the inequality

$$
l /(2 \cdot 5[(k+1) / 2])-3 n /\left(2 \cdot 12 k^{2}\right)<0 .
$$

Therefore $l \leqslant\left(15 n / 12 k^{2}\right)[(k+1) / 2]$. Hence by Lemma 12 ,

$$
r\left(L, C_{k}\right) \leqslant \frac{15 n}{12 k^{2}}\left[\frac{k+1}{2}\right]+2 k\left(\frac{15 n}{12 k^{2}}\left[\frac{k+1}{2}\right]+\left[n / 12 k^{2}\right]\right) \leqslant 2 n
$$

since $k \geqslant 3$. This gives a contradiction, which completes the proof.

Proposition 13 will be used in the proof of the following theorem. Just as we mentioned earlier, the bounds in Proposition 13 and Theorem 14 are not sharp.

THEOREM 14. Let $G$ be a connected graph with $n$ vertices and no more than $\left[n\left(1+1 / 42 k^{5}\right)\right]$ edges. Then for odd $k \geqslant 3$ and $n \geqslant 756 k^{10}$,

$$
r\left(G, C_{k}\right)=2 n-1 \text {. }
$$

Proof. The graph $K_{n-1} \cup K_{n-1}$ gives that $r\left(G, C_{k}\right) \geqslant 2 n-1$. Let $F$ be a graph on $2 n-1$ vertices whose complement $\bar{F}$ does not contain a $C_{k}$. We will show that $F$ contains $G$ as a subgraph.

Case I. $G$ has a suspended path with $\left[13 k^{5} / 2\right]+5(k+1) / 2+1$ vertices.

Let $H$ be the graph on $n-\left[13 k^{5} / 2\right]-1$ vertices obtained from $G$ by shortening the suspended path by $\left[13 k^{5} / 2\right]+1$ vertices. Then $r\left(H, C_{k}\right) \leqslant 2 n-1$ by Proposition 13. Therefore $F$ has $H$ as a subgraph. Let $H^{\prime}$ be the subgraph of $F$ which can be obtained from $H$ by lengthening the suspended path as much as possible (up to $\left[13 k^{5} / 2\right]+1$ vertices). If $H^{\prime}$ is $G$, the proof of this case is complete. If not, then Lemma liii) implies that $\bar{F}$ contains a path with $k$ vertices between any pair of vertices not in $H^{\prime}$. Hence any such pair of vertices must be adjacent in $F$. This gives that $G$ is a subgraph of $F$ since $F$ contains a complete graph on at least $n$ vertices.

Case II. $G$ has no suspended path with $\left[13 k^{5} / 2\right]+5(k+1) / 2+1$ vertices.

Lemma 2 implies that $G$ has at least

$$
n /\left(\left(\left[13 k^{5} / 2\right]+(k+1) / 2+1\right) 2\right)-3 n /\left(\left(42 k^{5}\right) 2\right)
$$

vertices of degree 1 . Since $n \geqslant 756 k^{10}, G$ has at least $\left(n / k^{5}\right)\left(\frac{1}{14}-\frac{1}{28}\right) \geqslant 27 k^{5}$ vertices of degree 1 . 
Let $H$ be the graph obtained from $G$ by deleting $13 k^{5}+(k-1) / 2$ vertices of degree 1. Proposition 13 implies that $r\left(H, C_{k}\right)<2 n-1$, and thus $H$ is a subgraph of $F$. Let $H^{\prime}$ be a maximal connected subgraph of $G$ containing $H$ which is a subgraph of $F$. If $H^{\prime}=G$, the proof is complete, so assume $H^{\prime}$ is a proper subgraph of $G$. Therefore there is a vertex $v$ of $H^{\prime}$ which is adjacent in $\bar{F}$ to each vertex not in $H^{\prime}$. Let $S$ be a subset of $V(F)-V\left(H^{\prime}\right)$ with $n$ vertices and let $R=V(F)-S$.

A $P_{k-1}$ in $\bar{F}$ using only the vertices of $S$ would imply that $C_{k}$ is a subgraph of $F$. If $k=3$, the result is trivial. Assume for the remainder of the proof that $k \geqslant 5$. Since $r\left(H, P_{k-1}\right) \leqslant n-(k-1) / 2$ by Proposition 5 , there is a copy of $H$ in $F$ using only vertices of $S$. Denote this copy of $H$ by $H_{1}$. By adding vertices of degree 1 enlarge $H_{1}$ as much as possible to obtain a subgraph $H_{1}^{\prime}$ of $F$ with $H_{1} \leqslant H_{1}^{\prime} \leqslant G$. If $H_{1}^{\prime}=G$ the proof is complete so assume not. Thus there is a vertex $v_{1}$ of $H_{1}^{\prime}$ and of $S$ which is adjacent in $\bar{F}$ to each vertex not in $H_{1}^{\prime}$. Let $N_{1}$ be the vertices adjacent to $v_{1}$ in $\bar{F}$. Hence $\left|N_{1}\right| \geqslant n$ and $\left|N_{1} \cap R\right| \geqslant n-13 k^{5}-(k-1) / 2$. By induction we will select $(k-1) / 2$ vertices from the set $S$. Assume $v_{1}, v_{2}, \ldots, v_{i}$ have been selected. Consider $S-\left\{v_{1}, v_{2}, \ldots, v_{i}\right\}$ and repeat the procedure for the selection of $v_{1}$ using $S-\left\{v_{1}, v_{2}, \ldots, v_{i}\right\}$ instead of $S$. This gives a vertex $v_{i+1}$. In this way one obtains a set $\left\{v_{1}, v_{2}, \ldots, v_{(k-1) / 2}\right\}$ of vertices of $S$ with corresponding sets $N_{i}(1 \leqslant i \leqslant(k-1) / 2)$, which satisfy $\left|N_{i}\right| \geqslant n$ and $\left|N_{i} \cap R\right| \geqslant n-13 k^{5}-$ $(k-1) / 2$.

$$
\begin{aligned}
& \text { Clearly } N=\cap_{i=1}^{(k-1) / 2}\left(N_{i} \cap R\right) \text { has at least } \\
& \qquad n-((k-1) / 2)\left(13 k^{5}+(k-1) / 2\right)
\end{aligned}
$$

vertices. Therefore there is a large complete bipartite graph in $\bar{F}$ since each vertex $v_{i}$ $(1 \leqslant i \leqslant(k-1) / 2)$ is adjacent in $\bar{F}$ to each vertex of $N$. In fact if $i \neq j, u_{i} \in N_{i}$, $u_{j} \in N_{j}$ and $u_{i} u_{j} \in E(\bar{F})$, then $\bar{F}$ contains a $C_{k}$ using $u_{i}, u_{j}$, and some vertices from $N$ and $\left\{v_{1}, v_{2}, \ldots, v_{(k-1) / 2}\right\}$. Thus we can assume that $u_{i} u_{j} \in E(F)$. This implies that $F$ contains a complete graph on the vertices of $N_{1} \cap N_{2}$ and each vertex of $N_{1}$ is adjacent in $F$ to each vertex in $N_{1} \cap N_{2}$. Since $\left|N_{1} \cap N_{2}\right|>n-27 k^{5}$ and $\left|N_{1}\right| \geqslant n, H$ is a subgraph of $F$. This completes the proof.

A tree is a special case of a sparse graph. Therefore we have the following corollary.

COROLlaRy 15. If $T_{n}$ is a tree with $n$ vertices, then for odd $k \geqslant 3$ and $n \geqslant 756 k^{10}$,

$$
r\left(T_{n}, C_{k}\right)=2 n-1 .
$$

It would be nice to know the smallest integer $n_{0}=n_{0}(k)$ such that if $n \geqslant n_{0}$ then $r\left(T_{n}, C_{k}\right)=2 n-1$.

\section{REFERENCES}

1. J. A. Bondy and P. Erdös, Ramsey numbers for cycles in graphs, J. Combin. Theory Ser. B 14 (1973), 46-54.

2. S. A. Burr, Generalized Ramsey theory for graphs-a survey, Graphs and Combinatorics, Lecture Notes in Math., vol. 406, Springer-Verlag, Berlin and New York, 1974, pp. 52-75.

3. ___ Ramsey numbers involving graphs with long suspended paths, J. London Math. Soc. (to appear). 
4. S. A. Burr, P. Erdös, R. J. Faudree, C. C. Rousseau and R. H. Schelp, An extremal problem in generalized Ramsey theory, Ars Combinatoria 10 (1980), 193-203.

5. V. Chvátal, Tree-complete graph Ramsey numbers, J. Graph Theory 1 (1977), 93.

6. R. J. Faudree, S. L. Lawrence, T. D. Parsons and R. H. Schelp, Path-cycle Ramsey numbers, Discrete Math. 10 (1974), 269-277.

7. L. Gerencsér and A. Gyárfas, On Ramsey-type problems, Ann. Univ. Sci. Budapest. Eötvös Sect. Math. 10 (1967), 67-70.

8. P. Hall, On representatives of subsets, J. London Math. Soc. 10 (1935), 26-30.

9. F. Harary, Graph theory, Addison-Wesley, Reading, Mass., 1969.

10. T. D. Parsons, Path-star Ramsey numbers, J. Combin. Theory Ser. B 17 (1974), 51-58.

Computer Science Department, City College (CUNY), New York, New York 10031 (Current address of S. A. Burr)

Department of Mathematics, Hungarian academy of Sciences, Budapest, Hungary (Current address of P. Erdös)

Department of Mathematical Sciences, Memphis State University, Memphis, Tennessee 38152 (Current address of R. J. Faudree, C. C. Rousseau and R. H. Schelp) 